\title{
Reduced contribution of thermally labile sugar lesions to DNA double strand break formation after exposure to heavy ions
}

\author{
Satyendra K Singh ${ }^{1}$, Alena Bencsik-Theilen', Emil Mladenov ${ }^{1}$, Burkhard Jakob², Gisela Taucher-Scholz ${ }^{2}$ \\ and George lliakis ${ }^{1 *}$
}

\begin{abstract}
In cells exposed to low linear energy transfer (LET) ionizing-radiation (IR), double-strand-breaks (DSBs) form within clustered-damage-sites (CDSs) from lesions disrupting the DNA sugar-phosphate backbone. It is commonly assumed that all DSBs form promptly and are immediately detected by the cellular DNA-damage-response (DDR) apparatus. However, there is evidence that the pool of DSBs detected by physical methods, such as pulsed-field gel electrophoresis (PFGE), comprises not only promptly forming DSBs (prDSBs) but also DSBs developing during lysis at high temperatures from thermally-labile sugar-lesions (TLSLS). We recently demonstrated that conversion of TLSLS to DNA breaks and ultimately to DSBs also occurs in cells during the first hour of post-irradiation incubation at physiological temperatures. Thus, TLSL-dependent DSBs (tIDSBs) are not an avoidable technique-related artifact, but a reality the cell always faces. The biological consequences of tIDSBs and the dependence of their formation on LET require in-depth investigation. Heavy-ions (HI) are a promising high-LET radiation modality used in cancer treatment. $\mathrm{HI}$ are also encountered in space and generate serious radiation protection problems to prolonged space missions. Here, we study, therefore, the effect of $\mathrm{HI}$ on the yields of tIDSBs and prDSBs. We report a reduction in the yield of tIDBSs stronger than that earlier reported for neutrons, and with pronounced cell line dependence. We conclude that with increasing LET the complexity of CDSs increases resulting in a commensurate increase in the yield prDSBs and a decrease in tIDSBs. The consequences of these effects to the relative biological effectiveness are discussed.
\end{abstract}

Keywords: DNA double strand breaks (DSB), lonizing radiation (IR), High LET, Heavy ions, Labile lesions, Radiation chemistry

\section{Introduction}

Ionizing radiation (IR) deposits energy as single ionizations or as ionization clusters that generate base and sugar damages in the DNA [1-3]. Clusters of ionization can generate clusters of DNA damage with different sizes and diverse damage composition (clustered damage sites, CDSs). Sugar damage can disrupt the sugar-phosphate backbone to generate DNA single-strand-breaks (SSBs) [2-4]. SSBs within CDSs form DNA double-strand-breaks (DSBs), which can have severe biological consequences [1,4-8].

\footnotetext{
* Correspondence: Georg.lliakis@uk-essen.de

${ }^{1}$ Institute of Medical Radiation Biology, University of Duisburg-Essen Medical School, Hufelandstr 55, Essen 45122, Germany

Full list of author information is available at the end of the article
}

DSBs can also be generated from CDSs populated with base damages through bi-stranded enzymatic opening during repair of the DNA sugar-phosphate backbone, or by combining with a SSB [7].

DSBs initiate rapid signaling and complex regulatory processes affecting DNA repair, cell cycle progression, transcription, translation, as well as decisions of programmed cell death and autophagy. These responses are currently integrated under the term cellular DNAdamage-response (DDR) [9].

Analysis of DDR after IR is based on the assumption that all DSBs form promptly. However, irradiation of plasmid DNA has shown that IR induces, in addition to sugar lesions promptly disrupting the sugar-phosphate backbone (prompt breaks), also lesions doing so after temperature-

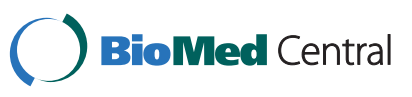


dependent chemical processing (delayed breaks) [10]. These thermally labile sugar lesions, TLSLs, constitute what are considered radiation-induced labile sites $[1,6,10]$. They can include diverse forms of sugar damage, abasic sites, and forms of base damage affecting sugar stability. Chemical evolution of such TLSLs to SSBs within a CDS can generate additional, TLSL-dependent DSBs (tlDSBs) [3,5,10-13].

Until recently, it was believed that in mammalian cells evolution of TLSLs to SSBs and the generation in this way of DSBs is only possible at high, non-physiological temperatures $\left(\sim 50^{\circ} \mathrm{C}\right)$ [14-16]. However, recent work from our laboratory $[17,18]$ provides evidence that IR induces TLSLs, which evolve within about $1 \mathrm{~h}$ under physiological temperatures to SSBs and contribute, when present within a CDS, to the formation of DSBs. These delayed-forming DSBs are thought to be generated continuously during the first postirradiation hour and to add to DSBs promptly induced (prDSBs). The biological consequences of tIDSBs remain to be elucidated, but are likely to be significant.

Since tIDBSs form within CDSs, the quality and quantity of which strongly depends on the linear energy transfer (LET) of the radiation employed, it is particularly important to study determinants of formation and rules of chemical tIDSBs processing after exposure to high LET radiation. First experiments along these lines using neutrons [19] showed a marked decrease in the yield of tlDBSs.

Heavy-ions (HI) are a promising high-LET radiation modality increasingly used in cancer treatment. Carbon ions are being used for the treatment of several types of solid cancers with promising results, and advanced treatment centers with ion accelerators are under construction in several countries. Important research issues related to the biological effects of $\mathrm{HI}$ and their relevance to the clinical application have been identified for in-depth investigation [20]. The nature of the DNA damage induced by HI and the contribution of tIDSBs to the biological effect is one such fundamental question.

$\mathrm{HI}$ are also encountered in space and generate serious radiation protection problems for long-duration missions to the earth's moon, or to Mars [21]. In preparing for such missions, the risk of cancer from space radiation must be estimated and mitigating measures must be developed. HI produce distinct forms of biological damage with largely unknown cancer risks. HI are therefore likely to require countermeasures different from those developed for low LET radiation [21]. Characterization of differences in the form of DNA damage generated by HI, particularly in the form of prDSBs and tlDSBs, is the first important step in this endeavor.

Here, we study the yields of tIDSBs and prDSBs in cells exposed to HI. We report a reduction in the yield of tlDBSs stronger than that earlier reported for neutrons [19], but still with pronounced cell line dependence.

\section{Materials and methods}

\section{Cell lines and culture conditions}

$M 059 K$, a repair proficient human glioma cell line and M059J its DNA-PKcs deficient counterpart [22], were grown in Dulbecco's Modified Eagle's Medium (D-MEM) supplemented with $10 \%$ fetal bovine serum (FBS), 1\% non-essential amino acids and 1\% L-glutamine. Mouse embryonic fibroblasts (MEFs) from $\mathrm{Lig}^{4^{-/-}} / \mathrm{p} 53^{-/-}$ and $\mathrm{Lig4}^{+/+} / p 53^{-/-}$mice [23] were grown in D-MEM supplemented with 10\% FBS and antibiotics. For experiments, cells were maintained in the exponential phase of growth at $37^{\circ} \mathrm{C}$ in a humidified incubator, in an atmosphere of $5 \% \mathrm{CO}_{2}$ and $95 \%$ air.

\section{Irradiation conditions for heavy ions $\left({ }^{58} \mathrm{Fe}\right)$ and $\mathrm{X}$-rays}

To analyze induction of DSBs, cells were resuspended in serum-free medium, and processed for PFGE as described earlier $[17,18]$.

Exposures to heavy ions (HI) were carried out at the GSI Helmholtzzentrum für Schwerionenforschung $\mathrm{GmbH}$ in Darmstadt, Germany. Typically cells were seeded in $25 \mathrm{~cm}^{2}$ tissue culture flasks and were incubated for $24 \mathrm{~h}$ at $37^{\circ} \mathrm{C}$ in Essen. The following day cells were transported in an insulated container filled with warm pads to maintain the temperature of the cells as close as possible to $37^{\circ} \mathrm{C}$, but without active heating. Upon arrival at the GSI, cells were promptly incubated at $37^{\circ} \mathrm{C}$ under standard growth conditions, and were allowed to recover for several hours from the transportation stress.

Cells were exposed to $1 \mathrm{GeV} / \mathrm{amu}$ heavy ions $\left({ }^{58} \mathrm{Fe}\right.$ or $\left.{ }^{62} \mathrm{Ni}\right)$. The particle LET under these conditions is $150 \mathrm{keV} / \mu \mathrm{m}$ and $175 \mathrm{keV} / \mu \mathrm{m}$ for ${ }^{58} \mathrm{Fe}$ and ${ }^{62} \mathrm{Ni}$, respectively. Dosimetry was carried out with a calibrated farmer chamber (PTW, Freiburg, Germany). The absolute particle fluence was measured with a calibrated ionization chamber (GSI, Darmstadt, Germany) at the beam exit window and the homogeneity of the scanned field was regularly checked using radiochromic EBT films (Ashland,Gafchromic, USA). The irradiation field was $5 \times 8 \mathrm{~cm}$ and was generated by multiple scanning of a pencil beam across the field with a dose deposition of 1 Gy per single scan. During irradiation cells were maintained at $4-8{ }^{\circ} \mathrm{C}$. Before exposure to heavy ions, tissue culture flasks were filled with growth media and were pre-cooled in ice-water for $15 \mathrm{~min}$ before placement in the irradiation holder. During the actual exposure to radiation, cells were not actively cooled. After radiation exposure, cells were transferred to the laboratory in ice. Where appropriate, irradiated cells were transferred to prewarmed $\left(45^{\circ} \mathrm{C}\right)$ media to quickly regenerate $37^{\circ} \mathrm{C}$ and start repair processes. The limited availability of $\mathrm{HI}$ for biological experiments made repeat-experiments impossible.

Control experiments were carried out by exposing cells to X-rays. In this case, irradiations were carried out also on ice with a Seifert-Pantak X-ray machine operated 
at $320 \mathrm{kV}, 10 \mathrm{~mA}$ with a $1.65 \mathrm{~mm} \mathrm{Al}$ filter (effective photon energy, $90 \mathrm{keV}$ ), at a dose rate of $3 \mathrm{~Gy} / \mathrm{min}$ and a distance of $50 \mathrm{~cm}$. Dosimetry was carried out using a calibrated ionization chamber and a chemical dosimeter. The mean LET of this type of radiation is, approximately, $2 \mathrm{keV} / \mu \mathrm{m}$.

\section{Pulsed-field gel electrophoresis (PFGE)}

In certain experiments, cells were lysed before irradiation using the low temperature lysis (LTL) protocol described below [15] and were incubated in TEN buffer (10 mM Tris- $\mathrm{HCl}, \mathrm{pH} 7.5,2 \mathrm{mM}$ EDTA, $50 \mathrm{mM} \mathrm{NaCl})$ to analyze TLSL-evolution. In other experiments, cells embedded in agarose blocks were irradiated in serumfree medium and were subsequently lysed by LTL.

In the standard, high temperature lysis (HTL) protocol [24], agarose blocks were placed in lysis buffer $(10 \mathrm{mM}$ Tris-HCl, pH 7.6, $50 \mathrm{mM} \mathrm{NaCl}, 10 \mathrm{mM}$ EDTA, 2\% Nlauryl sarcosyl, NLS, and $0.2 \mathrm{mg} / \mathrm{ml}$ protease added just before use) at $4{ }^{\circ} \mathrm{C}$ and were further processed at $50^{\circ} \mathrm{C}$ as described earlier $[17,18]$. Low temperature lysis (LTL) was carried out by maintaining samples below $4^{\circ} \mathrm{C}$ at all times using a published protocol [15], as described earlier [17,18].

Asymmetric, field-inversion gel electrophoresis (AFIGE) was carried out in gels cast with $0.5 \%$ molecular biology grade agarose (Bio-Rad) in the presence of $0.5 \mu \mathrm{g} / \mathrm{ml}$ ethidium bromide as described $[17,18]$. Gels were scanned using the "Typhoon" (GE-Healthcare) and the fraction of DNA released (FDR) from the well into the lane was quantified from images obtained using Image Quant 5.2 (GE-Healthcare).

\section{Treatment for the development of in-vitro of DSBs from TLSLs}

To monitor the kinetics of excess DSB formation at different temperatures in vitro, cells embedded in agarose blocks were exposed to IR, subjected to LTL, washed once for $1 \mathrm{~h}$ in TEN buffer $(0.5 \mathrm{ml} / \mathrm{plug})$ and the resulting agarose blocks containing the "naked" DNA were distributed to different tubes in the same buffer. Tubes were then transferred to water baths adjusted at different temperatures ranging between $10^{\circ} \mathrm{C}-50^{\circ} \mathrm{C}$ and incubated for different periods of time before washing once in $0.5 \mathrm{X}$ TBE and processing for PFGE.

\section{Results}

No detectable tIDSBs in some cell lines after exposure to HI

Previous work suggested that the contribution of TLSLs to the overall cellular DSB load decreases with increasing LET [19]. We inquired whether this trend persists with increasing LET of the radiation employed, as is the case for $\mathrm{HI}$ exposures. Figure 1A summarizes results obtained after exposure of the human tumor cell line, M059K, to iron ions $\left({ }^{58} \mathrm{Fe}\right)$. As previously reported $[17,18]$, exposure at $4^{\circ} \mathrm{C}$ of agarose-embedded M059K cells to different X-ray doses gives after lysis with the standard HTL protocol over $40 \%$ additional DSBs than lysis with the TLSL-preserving LTL protocol; these extra DSBs (tIDSBs) are generated by the thermal conversion within a CDS of TLSLs to SSBs.

Exposure of M059K cells to ${ }^{58} \mathrm{Fe}$ ions using the same experimental conditions shows higher yields of DSBs than X-rays after HTL, but interestingly no detectable modulation after analysis using LTL. This result indicates that the yields of tIDSBs approach zero in M059K cells exposed to ${ }^{58} \mathrm{Fe}$ ions.

Differences in the effectiveness at different endpoints between high and low LET radiations are conveniently compared by defining the relative biological effectiveness (RBE) as the quotient of the doses required for equal effect after exposure to X-rays and the test radiation modality. In the experiment shown in Figure $1 \mathrm{~A}$ and because of the practically linear dose-yield curves measured, this parameter can be determined in an effectindependent manner using the slopes of the resulting straight lines. Notably, widely different RBE values for ${ }^{58} \mathrm{Fe}$ versus X-ray exposure are calculated when using as basis the results obtained after HTL (reflecting the sum of prDSBs + tlDSBs) versus LTL (reflecting exclusively prDSBs). Specifically, a value of $\mathrm{RBE}_{\mathrm{HTL}}=1.12$ is calculated using the slopes of the $\mathrm{X}$-ray and ${ }^{58} \mathrm{Fe}$ dose-response curves after HTL, while a value of $\mathrm{RBE}_{\mathrm{LTL}}=1.91$ is obtained from the corresponding LTL data.

The response to ${ }^{58} \mathrm{Fe}$ noted above is reproduced in the DNA-PKcs deficient counterpart of M059K, the M059J cells $[25,26]$ (Figure 1B). The X-ray data reproduce again earlier findings $[17,18]$ and show that $45 \%$ more DSBs are detected after HTL as compared to LTL. On the other hand, after exposure to ${ }^{58} \mathrm{Fe}$, similar DSB yields are obtained after HTL and LTL. As a result $\mathrm{RBE}_{\mathrm{HTL}}=1.39$ and $\mathrm{RBE}_{\mathrm{LTL}}=2.70$ are calculated for the induction of DSBs in M059J cells.

We conclude that in a subgroup of cell lines, examples of which are M059K, and M059J, a contribution of TLSLs to excess DSB formation (tlDSBs) is marginal after exposure to ${ }^{58} \mathrm{Fe}$.

\section{Detectable formation of TLSL-dependent DSBs in some cell lines after exposure to ${ }^{58} \mathrm{Fe}$}

We noted before that the contribution of TLSL to DSBs is cell line specific $[17,18]$ and that this cell line specificity is also detectable after exposure to neutrons [19]. We explored therefore whether this also holds for exposures to ${ }^{58} \mathrm{Fe}$.

The results summarized in Figure 2A indicate a decrease by $63 \%$ in the number of DSBs after LTL as compared to HTL in Lig4 $^{-/-}$MEFs exposed to X-rays. Yet, a decrease by $39 \%$ is also registered after exposure of the same cells 

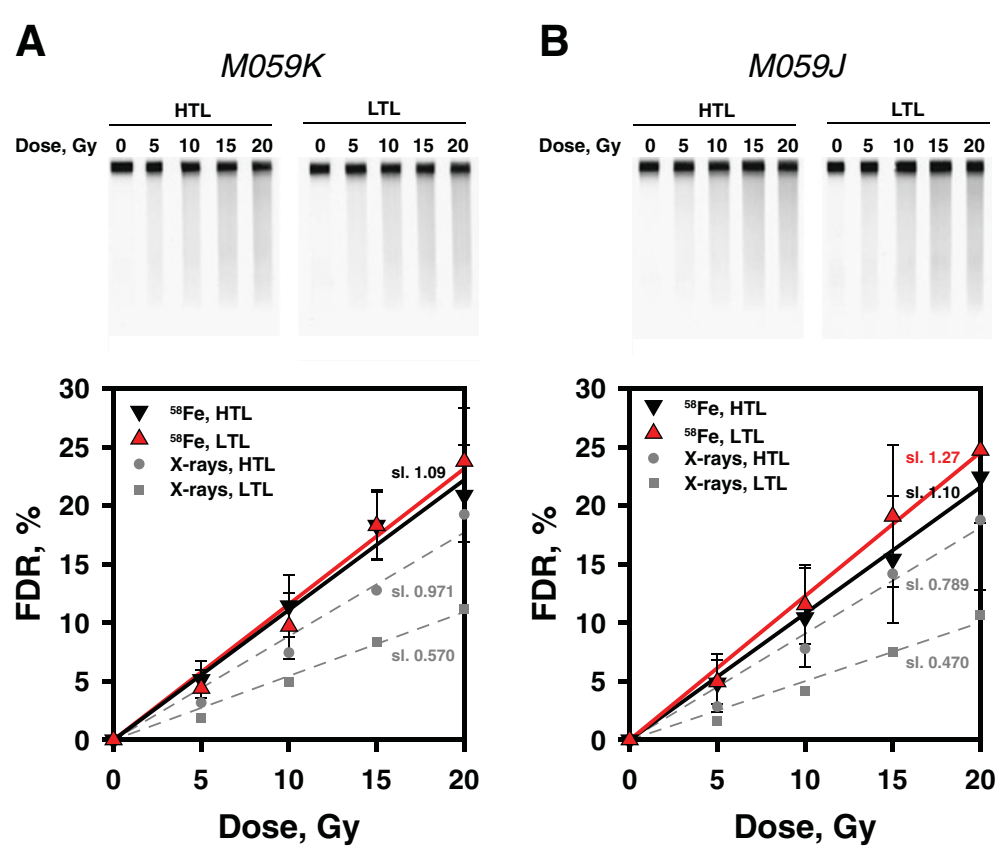

Figure 1 Dose response curves for the induction of DNA DSBs after ${ }^{58} \mathrm{Fe}$ ion irradiation in human M059K and M059J cells. Results earlier generated [19] with X-rays are depicted as gray symbols for comparison; they are similar to results generated in parallel to the ${ }^{58} \mathrm{Fe}$ irradiations but for limited number of doses in the range of interest (not shown). (A) Human M059K cells were embedded in agarose blocks and were exposed to different doses of $1 \mathrm{GeV}$ accelerated ${ }^{58} \mathrm{Fe}$ ions. Induction of DSBs was assayed by asymmetric field inversion gel electrophoresis (AFIGE), a pulsed-field gel electrophoresis method. Irradiated agarose blocks were processed immediately after irradiation using either HTL or LTL. Gels were scanned in a Fluor Imager and the fraction of DNA released from the wells into the lane (FDR) was determined for different radiation doses. Data represent the calculated average and standard deviation from 4 determinations (agarose blocks) in one experiment. (B) M059J cells embedded in agarose blocks were irradiated and analyzed as described above. Data represent the calculated average and standard deviation from 4 determinations in one experiment.

to ${ }^{58} \mathrm{Fe}$ indicating a significant contribution of TLSLs to the formation of DSBs. Here, an $\mathrm{RBE}_{\mathrm{HTL}}=1.12$ and an $\mathrm{RBE}_{\mathrm{LTL}}=1.83$ are calculated for the induction of DSBs after exposure to ${ }^{58} \mathrm{Fe}$.

We conclude that while the contribution of TLSLs to excess DSB formation is reduced after exposure to ${ }^{58} \mathrm{Fe}$, the level of this reduction is cell line dependent.

It may be relevant to mention here that small DNA fragments, undetectable by PFGE, are produced in higher yields after exposure to high, as compared to low, LET radiation. Thus, the yields of DSBs measured after exposure to high LET radiation are likely to be underestimated. However, we consider unlikely that this inherent limitation in the detection of DSBs compromises our conclusions.

\section{Different yields of TLSL-dependent DSBs after exposure to ${ }^{58} \mathrm{Fe}$ of naked DNA and chromatin}

To further confirm the absence of TLSL induced DSBs in ${ }^{58} \mathrm{Fe}$ exposed M059K cells (Figure 1A), we exposed agarose-embedded cells to 20 Gy and processed them immediately by LTL to obtain agarose-embedded, "naked" DNA in which radiation-induced lesions, including TLSLs, were preserved [18]. In these agarose blocks, TLSL stability can be studied through their contribution to DSB formation after in-vitro incubation at different temperatures.

The results summarized in Figure 2B show no significant increase in FDR for incubations in TEN-buffer at temperatures between 4 and $50^{\circ} \mathrm{C}$ for up to $48 \mathrm{~h}$. Similar experiments carried out with cells exposed to X-rays show large increases in FDR for post-lysis incubations at temperatures above $20^{\circ} \mathrm{C}$ [18]. The lack of excess DSB formation following incubation at high temperatures of DNA from ${ }^{58} \mathrm{Fe}$-exposed M059K cells is in-line with the similar dose-response curves shown in Figure 1A following HTL and LTL.

Collectively, the above results suggest that cell line specific biochemical parameters contribute to the generation of tlDSBs, even after exposure to high LET radiation. To begin characterizing parameters defining this effect, we used LTL to lyse non-irradiated M059K cells and exposed the resulting agarose-embedded "naked" DNA to 5 Gy of ${ }^{62} \mathrm{Ni}$. In this way, a variety of lesions, including TLSLs, are generated in naked DNA kept in a defined buffer. This condition is biochemically better characterized than irradiation of chromatin organized DNA in the cellular environment. 


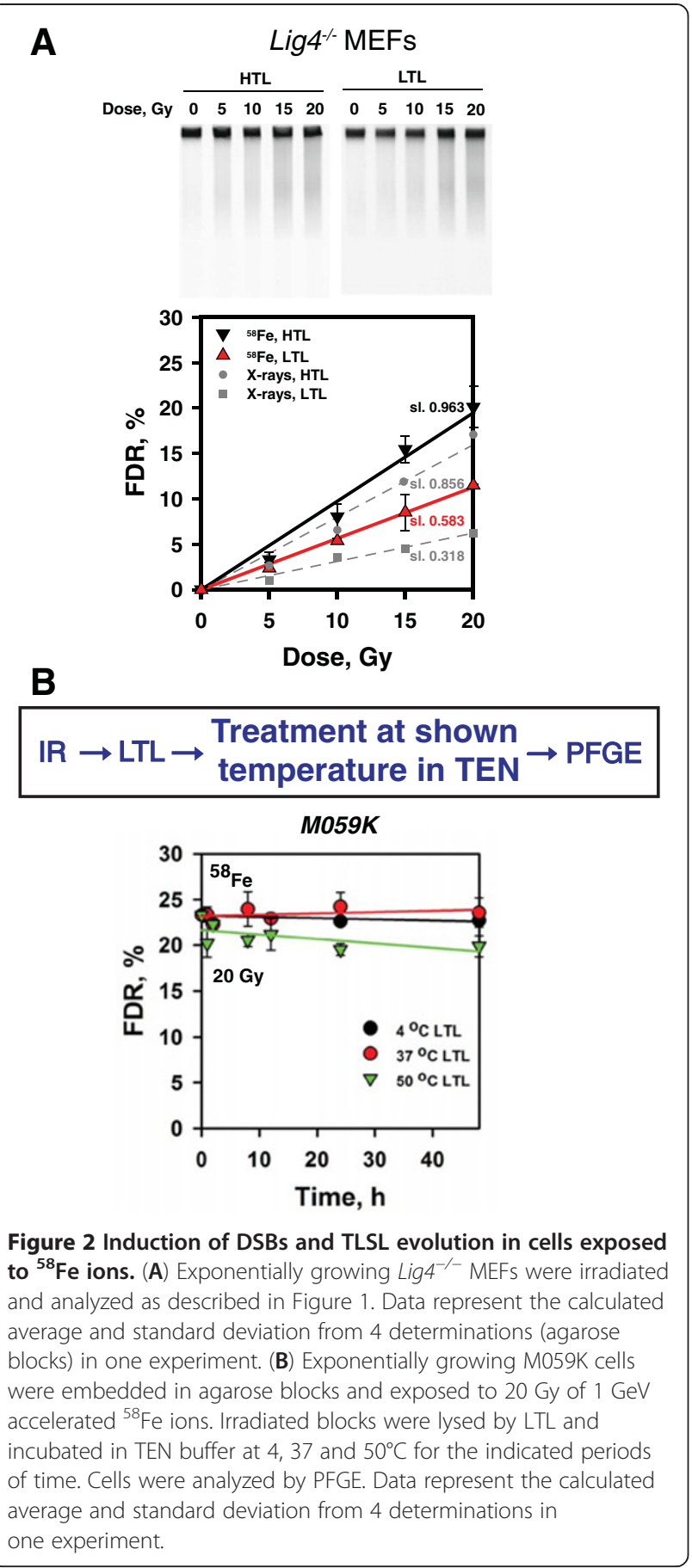

Agarose blocks generated in this manner were transferred to TEN buffer and after irradiation were incubated at different temperatures for different periods of time. The results obtained are summarized in Figure 3A and show that ${ }^{62} \mathrm{Ni}$ ions generate TLSLs in "naked" DNA that readily convert to DSBs after incubation at temperatures between $37-50^{\circ} \mathrm{C}$, with kinetics similar to that measured after exposure to X-rays [18]. Thus, DNA organization is a key determinant of the chemical characteristics and the associated thermal stability of tIDSBs not only after exposure to low LET but also after exposure to high LET radiation.

We inquired whether indirect radiation effects by water radical production underpin the TLSL-dependent formation of excess DSBs after incubation at high temperatures. For this purpose we carried out the "naked"-DNA experiment described above in the presence of $2 \%$ DMSO, an effective scavenger of ${ }^{\circ} \mathrm{OH}$ radicals. The results summarized in Figure $3 \mathrm{~B}$ indicate that when irradiation of naked DNA is carried out in the presence of DMSO, subsequent incubation at high temperatures reduces the yields of excess DSBs generated, pointing to a contribution of ${ }^{\circ} \mathrm{OH}$ in the production of TLSLs. We conclude that ${ }^{\circ} \mathrm{OH}$ has an essential contribution to the generation of tDSBs when

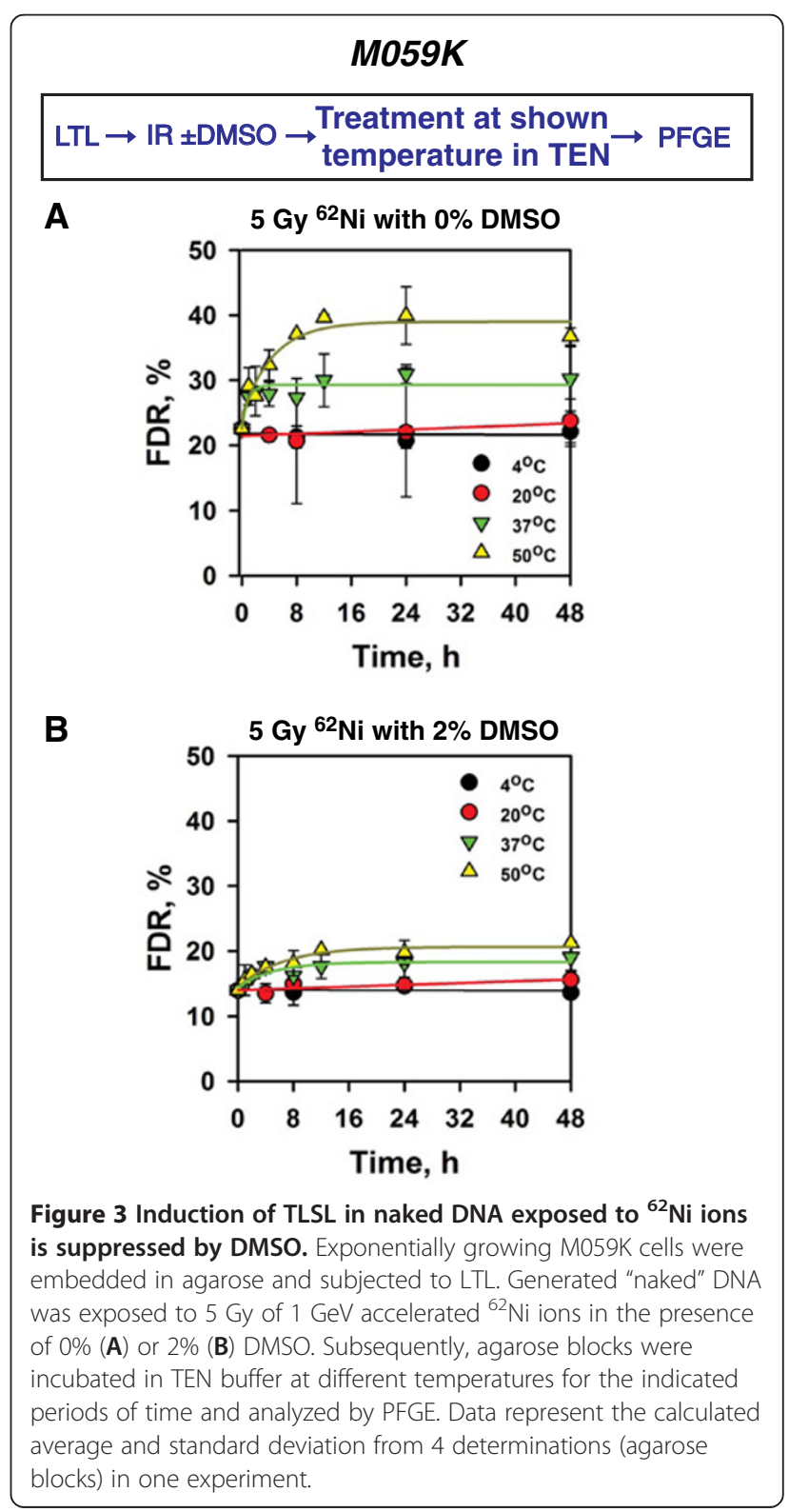


irradiating "naked" DNA. This result cannot be directly extended to cell irradiation because the chromatin organization of the DNA is protecting it from ${ }^{\circ} \mathrm{OH}$ attacks.

\section{Processing of DSBs by NHEJ is slower after exposure to HI than X-rays}

In wild-type M059K cells, total DSBs (analyzed by HTL) induced by $1 \mathrm{GeV} / \mathrm{amu}{ }^{58} \mathrm{Fe}$ are repaired with nearly the same efficiency as those induced by X-rays (Figure 4A); however, more unrepaired DSBs are detected between 2 $8 \mathrm{~h}$ after exposure to $\mathrm{HI}$. Within statistical variation, a similar response is also observed when DSB repair kinetics is measured by LTL to specifically assay for prDSBs (Figure 4B). Similar overall trends are also obtained when analyzing wild type MEFs exposed to ${ }^{62} \mathrm{Ni}$ ions (Additional file 1: Figure S1).

In DNA-PKcs deficient M059J cells (Figure 5A), where D-NHEJ is defective and repair of DSBs is mainly mediated by B-NHEJ [27-29], repair of ${ }^{58} \mathrm{Fe}$-induced total DSBs is compromised slightly stronger than in M059K cells. Thus, the increased DSB complexity of ${ }^{58} \mathrm{Fe}$ generated DSBs appears to compromise processing by B-NHEJ to a greater extent than processing by D-NHEJ.

When DSB repair kinetics is measured in M059J cells using LTL to focus analysis on prDSBs and tlDSBs forming during repair, complex kinetics is observed after exposure to X-rays (Figure 5B): The load of DSBs rises at early times and decays subsequently. This structure derives from the fact that the kinetics reflects not only the processing of prDBSs by B-NHEJ, but also the gradual development and subsequent processing of tIDSBs (see [18] for a more in-depth analysis of the components involved). It is not detectable in repair proficient M059K cells, either owing to the higher efficiency of DSB repair, or to differences in the kinetics of tlDSB production.

Repair kinetics after exposure to ${ }^{58} \mathrm{Fe}$ ions lacks this structure, in line with the observation that only few tIDSBs are produced in M059J cells after HI radiation (see Figure 1B). We do not have an explanation why similar levels of residual damage is observed after $2 \mathrm{~h}$ in cells exposed to X-rays and ${ }^{58} \mathrm{Fe}$ when using LTL, but this may reflect analysis artifacts. These may originate from shifts in the yields of prDSBs and tDSBs with increasing LET, as well as from the normalization applied (to show percent of initial damage). Qualitatively similar results are also obtained after exposure of $\mathrm{Lig4^{-/- }}$ MEFs to ${ }^{62} \mathrm{Ni}$ ions (Additional file 2: Figure S2). However, with these cells and probably as a consequence of the significant induction of tlDSBs after exposure to HI (Figure 2A), repair of ${ }^{62} \mathrm{Ni}$ induced DSBs is compromised as compared to X-rays when analyzed by LTL (Additional file 2: Figure S2B).

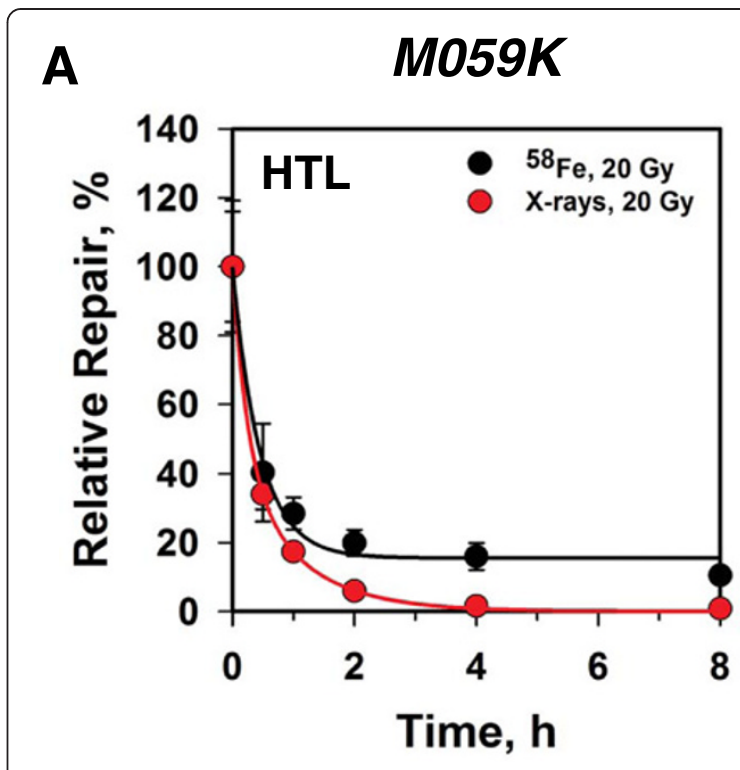

B

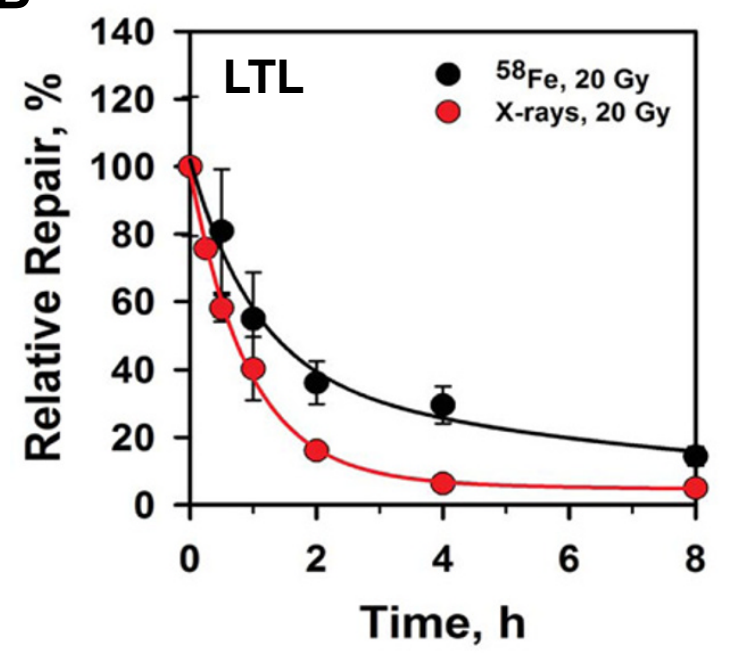

Figure 4 DSB repair kinetics of human M059K cells exposed to ${ }^{58} \mathrm{Fe}$ ions or X-rays. (A) Exponentially growing M059K cells were exposed to $20 \mathrm{~Gy}$ of $\mathrm{X}$-rays or ${ }^{58} \mathrm{Fe}$ ions and returned to standard incubation conditions for repair. Agarose blocks were prepared and lysed by HTL (A) or LTL (B) before processing by PFGE. Plotted is relative repair as a function of repair time. To calculate relative repair, FDR at different time points was divided by the FDR measured at the $0 \mathrm{~h}$ time point (this value was obtained from the dose response curve). Data represent the calculated average and standard deviation from 4 determinations in one experiment.

In an effort to connect the above observations on the induction and repair of different forms of DSBs with the cell inactivation potential of $\mathrm{HI}$, cell survival was determined. The results obtained with M059K and M059J cells are summarized in Figure 6. Shown in the figure for comparison are also results previously reported with the same cell lines after exposure to X-rays, in the presence or absence of $10 \mu \mathrm{M}$ wortmannin to inhibit D- 


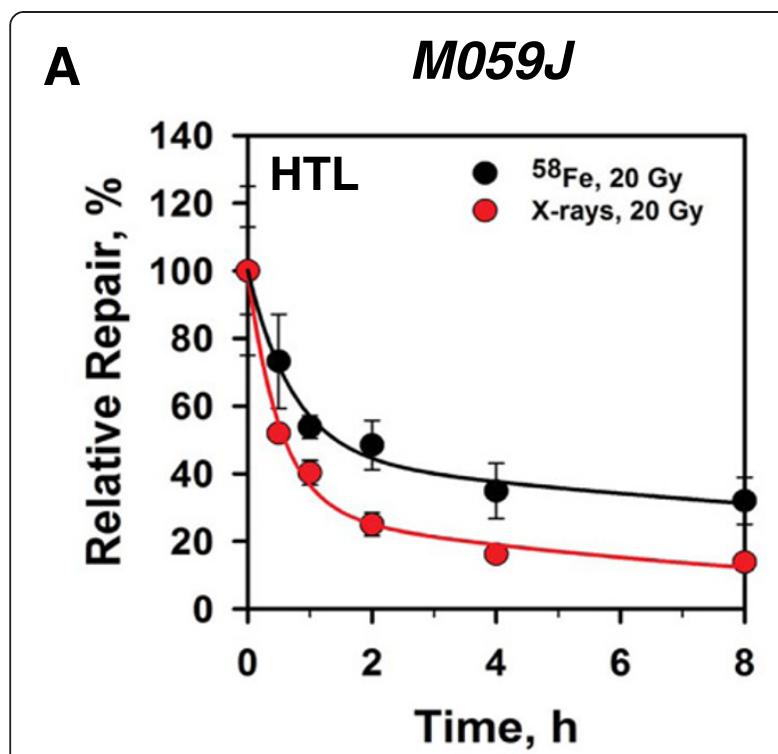

B

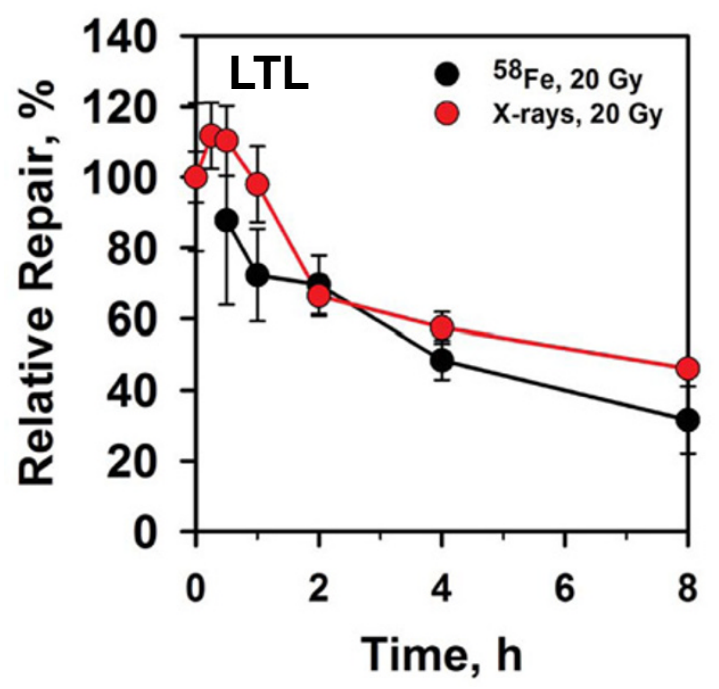

Figure 5 DSB repair kinetics of human M059J cells exposed to ${ }^{58} \mathrm{Fe}$ ions or X-rays. Other details as in Figure 4.

NHEJ [24]. M059K cells exposed to X-rays show the typical dose response, characterized by a shoulder at low radiation doses followed by an exponential region at higher doses. Wortmannin-induced inhibition of D-NHEJ strongly sensitizes M059K cells to X-rays and leads to an exponential survival curve. Notably, M059K cells exposed to ${ }^{58} \mathrm{Fe}$ ions show cell survival practically indistinguishable from that of wortmannin-treated cells. From these results RBEs of 3.8, 2.5, and 2.3 are calculated at surviving fractions of $0.1,0.01$, and 0.001 , respectively.

M059J cells, as a result of their deficiency in D-NHEJ, are intrinsically highly radiosensitive and wortmannin has no further radiosensitizing effect. Notably, ${ }^{58} \mathrm{Fe}$ ions are killing these cells with efficiency practically indistinguishable from

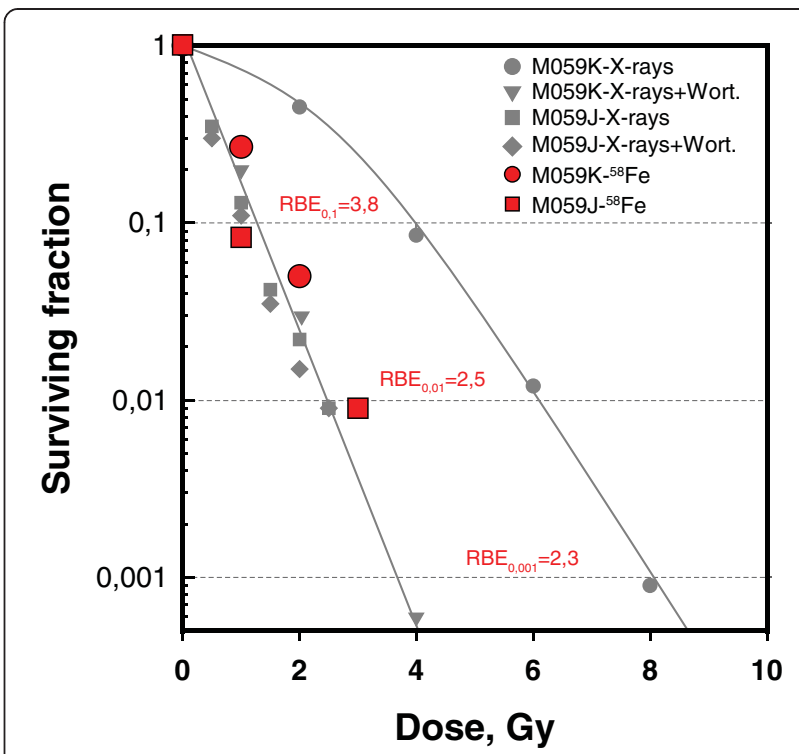

Figure 6 Survival curves of logarithmically growing M059K and M059J cells as measured by colony formation after exposure to $1 \mathrm{GeV}^{58} \mathrm{Fe}$ ions (red symbols). Shown (gray symbols) for comparison are also results of experiments previously reported [24] with these cells after exposure to $\mathrm{X}$-rays in the presence or absence of $20 \mu \mathrm{M}$ wortmannin. The figure also shows RBE values calculated at the indicated levels of cell survival. The lines drawn follow the early data points [24] and are given as basis for comparisons with the results generated with ${ }^{58} \mathrm{Fe}$.

that of X-rays, which leads to RBEs of approximately 1 . This observation is in line with earlier reports pointing to a D-NHEJ proficiency requirement for high LET mediated radiosensitization [30-33].

\section{Discussion}

There is evidence for the induction by IR of thermally labile DNA lesions, which contribute to DSB formation (tlDSBs), albeit in a delayed manner, even in cells maintained under physiological temperatures (see Introduction). As a result of this delayed formation, the total load of DSBs generated in an irradiated cell (tDBSs) will be the sum of those induced promptly, i.e. those present immediately after irradiation (prDBSs), and those generated within a non-DSB-CDS by the conversion of a TLSL to a SSB (tlDBSs); thus, $\mathrm{tDSBs}=$ prDBSs + tlDBSs. It is not known whether prDBSs and tIDBSs are detected and processed by the cell with the same efficiency and, actually, arguments can be developed why this may not be the case [17-19]. If cells detect and process differently prDBSs and tlDBSs, it is likely that their biological consequences will also be different.

Experimentally, the yields of prDBSs can be determined by lysing cells immediately after irradiation using low temperature $\left(0-4^{\circ} \mathrm{C}\right)$ lysis protocols $(\mathrm{LTL})$, whereas the standard $50^{\circ} \mathrm{C}$ lysis allows determination of tDBSs. The difference between tDBSs - prDBSs yields gives then 
estimates regarding the yields of tlDBSs. There is evidence that IR induces a spectrum of TLSLs with different levels of chemical and thermal stability [18]. This raises the question how to determine the biologically relevant subset of tlDBSs, i.e. the subset that also converts to a DSB in cells maintained under physiological conditions. There are at present no established methods allowing the reliable determination of the biologically relevant subset of tIDBSs. However, as a first approximation, we assume that conversion of TLSLs to breaks is similar in cells maintained at $37^{\circ} \mathrm{C}$ and cells analyzed by lysing at $50^{\circ} \mathrm{C}$ immediately after exposure to IR [18].

Using the above outlined conceptual and experimental background we investigate here how the yields of prDBSs and tIDBSs change in cells exposed to HI. The results presented in the previous section extend trends previously reported for neutrons [19] and confirm a strong, inverse LET dependence of the yields of prDBSs and tIDBSs. Specifically, while exposure to HI causes a strong reduction in the yields of tlDBSs as compared to X-rays, it causes a strong increase in the yields of prDBSs. These opposing effects partly compensate each other and as a result the yield of tDSBs changes only modestly with increasing LET. This is in line with the observation that RBEs close to 1 are frequently measured for the induction of DSBs [34]. Notably, our results demonstrate that when prDBSs are specifically detected by LTL protocols, much higher RBE values are measured that are approaching those obtained for cell survival (Figure 6). This is a potentially highly significant observation that warrants further investigations.

Notably, the RBE values for DSB-induction after exposure to high LET radiation, as measured by $\gamma-\mathrm{H} 2 \mathrm{AX}$ foci formation in diverse cell lines, is also very close to one ([34] and references therein). This is significant as it shows, in line with our earlier work [18], that the load of DSBs the cell ultimately "sees" is close to that measured by HTL. If cells were only detecting prDBSs, as it is often assumed, two to three times more DSBs (i.e. $\gamma$-H2AX foci) would have been expected after exposure to high LET radiation than after low LET radiation. On the other hand, it also demonstrates that the $\gamma-\mathrm{H} 2 \mathrm{AX}$ marking of DSBs does not differentiate levels of DSB complexity.

The increase in prDSBs observed with increasing LET can be explained by the expected increase in the size of ionization clusters (more ionizations within the same volume) that leads to the generation of higher complexity CDS, i.e. the presence of a higher number of lesions at the site. As a result of this increase in lesion number within a CDS it becomes more likely that prompt SSBs will combine to form a prDSB. Even if TLSLs are present in these CDSs, their subsequent conversion to breaks will remain inconsequential with reference to DSB formation. The chemical reactions that convert a TLSL to a SSB remain uncharacterized, but may include base-catalyzed hydrolysis or oxidation [19].

As noted above, TLSLs are not a uniform chemical entity but rather a spectrum of lesions with different chemical and thermal sensitivities. The probability of their formation from clusters of ionization events and radical attacks, as well as their chemical evolution may be decisively determined by the chemical environment in their immediate vicinity. In this respect, it is likely that the details of DNA organization in chromatin within a CDS, including all participating histone and non-histone proteins, will affect decisively not only the induction of TLSLs but also their evolution to tlDBSs. This theoretically anticipated dependence provides also a first explanation for the surprisingly large differences observed in the yields of tIDBSs among different cell lines both after exposure to high as well as to low LET radiation [17-19]. Furthermore, the large differences in LTL dose response curves among different cell lines contrasts the surprisingly similar HTL dose response curves and points to cell line specific variation in the chemical environment in the vicinity of a clustered ionization hitting the DNA that alters the probability of generation of a prDSB.

While the selection of cell lines used here reflects the intellectual evolution of the TLSL problematic in our work during the past few years, future work will certainly benefit from a hypothesis oriented selection of cell lines and an analysis of TLSL production and evolution after treatments that alter chromatin organization. It is also worth pointing out that the differences between cell lines persist even after exposure of cells to high LET radiation [19] (see above).

The results discussed above demonstrate that the energy deposition pattern of radiation is not the sole determinant of the yields of tlDSBs. Small differences in DNA organization may cause changes in the induction and the subsequent chemical processing of TLSLs and may strongly affect the form of DSBs induced - even after exposure to high LET radiation. Worth noting is also that the differences observed in lesion induction and evolution among cell lines exposed to $\mathrm{HI}$ are largely eliminated if "naked" DNA is irradiated instead of cells.

Collectively, the results presented here, as well those published before [17-19], point to an unexplored dimension in the production of DNA damage by IR. Temporal evolution of complex radiation damage to DSBs, and the suggested role of DNA organization in this evolution go beyond current concepts of DNA damage induction and repair [35] and indicate aspects of DDR that warrant further investigations.

It is tempting to speculate that transient, chemical stabilization of TLSLs, may allow repair of SSBs and base damages within a non-DSB CDS, so that subsequent conversion of the TLSL to a DNA break will not cause a DSB. 
Such agents may find application in radiation protection on earth and in space, as well as in the development of new strategies in radiation oncology $[21,36,37]$.

\section{Additional files}

Additional file 1: Figure S1. Repair kinetics of exponentially growing wild type MEFs after exposure to $1 \mathrm{GeV}^{62} \mathrm{Ni}$ ions or $\mathrm{X}$-rays. Other details as in Figure 4.

Additional file 2: Figure S2. Repair kinetics of exponentially growing $\mathrm{Lig4}^{-1-}$ MEFs after exposure to $1 \mathrm{GeV}^{62} \mathrm{Ni}$ ions or X-rays. Other details as in Figure 4.

\section{Competing interests}

The authors declare that they have no competing interests.

\section{Authors' contributions}

SKS designed and performed the PFGE experiments, analyzed and interpreted the results obtained and contributed to the preparation of the manuscript. ABT designed and performed the cell survival experiments. EM performed cell survival experiments and helped in the preparation of the manuscript. BJ and GTS helped design the irradiations with heavy ions at the GSI and assisted in the interpretation of the results obtained, as well as in the preparation of the manuscript. Gl conceived the project, assisted in the experimental design, interpreted the results obtained and wrote the manuscript. All authors read and approved the final manuscript.

\section{Acknowledgments}

Work supported by grants from the "Bundesministerium für Wirtschaft und Technologie"(BMWi: ESA-AO-08-IBER, 50WB1229) and the „Bundesministerium für Bildung und Forschung"(BMBF: 02NUK005C and 03NUK001B). The authors are indebted to Fred Alt and Joan Allalunis-Turner for cells.

\section{Author details}

${ }^{1}$ Institute of Medical Radiation Biology, University of Duisburg-Essen Medical School, Hufelandstr 55, Essen 45122, Germany. ${ }^{2}$ GSI Helmholtzzentrum für Schwerionenforschung GmbH, Division of Biophysics, Darmstadt, Germany.

Received: 20 December 2012 Accepted: 22 March 2013

Published: 2 April 2013

\section{References}

1. Goodhead DT: Initial events in the cellular effects of ionizing radiations: clustered damage in DNA. Int J Radiat Biol 1994, 65:7-17.

2. von Sonntag C: The chemical basis of radiation biology. London - New York Philadelphia: Taylor \& Francis; 1987.

3. von Sonntag C: Free-radical-induced DNA damage and Its repair. Berlin Heidelberg: Springer; 2006.

4. Ward JF: Biochemistry of DNA lesions. Radiat Res 1985, 104:S103-S111.

5. Ward JF: DNA damage produced by ionizing radiation in mammalian cells: identities, mechanisms of formation, and reparability. Prog Nucl Acid Res 1988, 35:95-125.

6. Brenner DJ, Ward JF: Constraints on energy deposition and target size of multiply damaged sites associated with DNA double-strand breaks. Int J Radiat Biol 1992, 61:737-748.

7. Georgakilas AG: Processing of DNA damage clusters in human cells: current status of knowledge. Mol Biosyst 2008, 4:30-35.

8. O'Neill P, Wardman P: Radiation chemistry comes before radiation biology. Int J Radiat Biol 2009, 85:9-25.

9. Jackson SP, Bartek J: The DNA-damage response in human biology and disease. Nature 2009, 461:1071-1078.

10. Jones GD, Boswell TV, Ward JF: Effects of postirradiation temperature on the yields of radiation-induced single- and double-strand breakage in SV40 DNA. Radiat Res 1994, 138:291-296.

11. Lafleur MVM, Woldhuis J, Loman H: Alkali-labile sites and post-irradiation effects in gamma-irradiated biologically active double-stranded DNA in aqueous solution. Int J Radiat Biol 1979, 36:241-247.
12. Henle ES, Roots R, Holley WR, Chatterjee A: DNA strand breakage is correlated with unaltered base release after gamma irradiation. Radiat Res 1995, 143:144-150

13. Fung $H$, Demple B: Distinct roles of Ape1 protein in the repair of DNA damage induced by ionizing radiation or bleomycin. J Biol Chem 2011, 286:4968-4977.

14. Rydberg B: Radiation-induced heat-labile sites that convert into DNA double-strand breaks. Radiat Res 2000, 153:805-812

15. Stenerlöw B, Karlsson KH, Cooper B, Rydberg B: Measurement of prompt DNA double-strand breaks in mammalian cells without including heatlabile sites: results for cells deficient in nonhomologous end joining. Radiat Res 2003, 159:502-510.

16. Karlsson $\mathrm{KH}$, Stenerlöw B: Extensive ssDNA end formation at DNA doublestrand breaks in non-homologous end-joining deficient cells during the S phase. BMC Mol Biol 2007, 8:97.

17. Singh SK, Wu W, Wu W, Wang M, lliakis G: Extensive repair of DNA doublestrand breaks in cells deficient in the DNA-PK dependent pathway of NHEJ after exclusion of heat-labile sites. Radiat Res 2009, 172:152-164.

18. Singh SK, Wang M, Staudt C, lliakis G: Post-irradiation chemical processing of DNA damage generates double-strand breaks in cells already engaged in repair. Nucleic Acids Res 2011, 39:8416-8429.

19. Singh SK, Wu W, Stuschke M, Bockisch A, lliakis G: Reduced contribution of thermally-labile sugar lesions to DNA double-strand break formation after exposure to neutrons. Radiat Res 2012, 178:581-590.

20. Durante M, Loeffler JS: Charged particles in radiation oncology. Nat Rev Clin Oncol 2010, 7:37-43.

21. Durante $M$, Cucinotta FA: Heavy ion carcinogenesis and human space exploration. Nat Rev Cancer 2008, 8:465-472.

22. Allalunis-Turner MJ, Zia PKY, Barron GM, Mirzayans R, Day RS III: Radiationinduced DNA damage and repair in cells of a radiosensitive human malignant glioma cell line. Radiat Res 1995, 144:288-293.

23. Frank KM, Sharpless NE, Gao Y, Sekiguchi JM, Fergson DO, Zhu C, Manis JP, Horner J, DePinho RA, Alt FW: DNA ligase IV deficiency in mice leads to defective neurogenesis and embryonic lethality via the p53 pathway. Mol Cell 2000, 5:993-1002.

24. DiBiase SJ, Zeng Z-C, Chen R, Hyslop T, Curran WJ Jr, lliakis G: DNAdependent protein kinase stimulates an independently active, nonhomologous, end-joining apparatus. Cancer Res 2000, 60:1245-1253.

25. Allalunis-Turner MJ, Barron GM, Day RS, Dobler KD, Mirzayans R: Isolation of two cell lines from a human malignant glioma specimen differing in sensitivity to radiation and chemotherapeutic drugs. Radiat Res 1993, 134:349-354.

26. Lees-Miller SP, Godbout R, Chan DW, Weinfeld M, Day RS III, Barron GM, Allalunis-Turner J: Absence of p350 subunit of DNA-activated protein kinase from a radiosensitive human cell line. Science 1995, 267:1183-1185.

27. Iliakis G: Backup pathways of NHEJ in cells of higher eukaryotes: cell cycle dependence. Radiother Oncol 2009, 92:310-315.

28. Iliakis G, Wu W, Wang M, Terzoudi Gl, Pantelias GE: Backup pathways of nonhomologous End joining May have a dominant role in the formation of chromosome aberrations. In Chromosomal alterations. Edited by Obe G. Vijayalaxmi. Berlin, Heidelberg, New York: Springer Verlag; 2007:67-85. Repair/NHEJ/Reviews SD (Series Editor).

29. Niakis G, Wang H, Perrault AR, Boecker W, Rosidi B, Windhofer F, Wu W, Guan J, Terzoudi G, Pantelias G: Mechanisms of DNA double strand break repair and chromosome aberration formation. Cytogenet Genome Res 2004, 104:14-20.

30. Zyuzikov NA, Prise KM, Zdzienicka MZ, Newman HC, Michael BD, Trott KR: The relationship between the RBE of alpha particles and the radiosensitivity of different mutations of chinese hamster cells. Radiat Environ Biophys 2001, 40:243-248.

31. Hill MA, Herdman MT, Stevens DL, Jones NJ, Thacker J, Goodhead DT: Relative sensitivities of repair-deficient mammalian cells for clonogenic survival after a-particle irradiation. Radiat Res 2004, 162:667-676.

32. Wang $H$, Wang $X$, Zhang $P$, Wang $Y$ : The Ku-dependent non-homologous end-joining but not other repair pathway is inhibited by high linear energy transfer ionizing radiation. DNA Repair (Amst) 2008, 7:725-733.

33. Li Y, Qian H, Wang Y, Cucinotta FA: A stochastic model of DNA fragments rejoining. PLoS One 2012, 7:e44293.

34. Franken NA, Hovingh S, Ten Cate R, Krawczyk P, Stap J, Hoebe R, Aten J, Barendsen GW: Relative biological effectiveness of high linear energy transfer a-particles for the induction of DNA-double-strand breaks, chromosome aberrations and reproductive cell death in SW-1573 lung tumour cells. Oncol Rep 2012, 27:769-774. 
35. Jeggo PA, Geuting V, Löbrich M: The role of homologous recombination in radiation-induced double-strand break repair. Radiother Oncol 2011, 101:7-12.

36. Newhauser WD, Durante M: Assessing the risk of second malignancies after modern radiotherapy. Nat Rev Cancer 2011, 11:438-448.

37. Cucinotta F, Durante M: Cancer risk from exposure to galactiv cosmic rays: implications for space exploration by humans beings. Lancet Oncol 2006, 7:431-435.

doi:10.1186/1748-717X-8-77

Cite this article as: Singh et al:: Reduced contribution of thermally labile sugar lesions to DNA double strand break formation after exposure to heavy ions. Radiation Oncology 2013 8:77.

\section{Submit your next manuscript to BioMed Central and take full advantage of:}

- Convenient online submission

- Thorough peer review

- No space constraints or color figure charges

- Immediate publication on acceptance

- Inclusion in PubMed, CAS, Scopus and Google Scholar

- Research which is freely available for redistribution 